\title{
Assessment of Multi-Temporal Image Fusion for Remote Sensing Application
}

\author{
Yuhendra", Joshapat Tetuko Sri Sumantyo* \\ ${ }^{*}$ Department of Informatics, Faculty of Engineering, Padang Institute of Technology, Indonesia \\ E-mail: yuhendrasan@gmail.com \\ * Josaphat Microwave Remote Sensing Laboratory (JMRSL), Chiba University, Japan \\ E-mail: jtetuko@chiba-u.ac.jp
}

\begin{abstract}
Image fusion and subsequent scene analysis are important for studying Earth surface conditions from remotely sensed imagery. The fusion of the same scene using satellite data taken with different sensors or acquisition times is known as multi-sensor or multi-temporal fusion, respectively. The purpose of this study is to investigate the effects of misalignments the multi-sensor, multitemporal fusion process when a pan-sharpened scene is produced from low spatial resolution multispectral (MS) images and a high spatial resolution panchromatic (PAN) image. It is found that the component substitution (CS) fusion method provides better performance than the multi-resolution analysis (MRA) scheme. Quantitative analysis shows that the CS-based method gives a better result in terms of spatial quality (sharpness), whereas the MRA-based method yields better spectral quality, i.e., better color fidelity to the original MS images.
\end{abstract}

Keywords—multi-sensor; multi-temporal fusion; component substitution; multi-resolution analysis

\section{INTRODUCTION}

Following the rapid advancements of new and greatly improved remote sensing (RS) sensor systems, various kinds of remote sensing data were acquired and applied in many interdisciplinary Earth observational applications. Remote sensing images are characterized by their spectral, spatial, radiometric, and temporal resolutions. Spectral resolution refers to the bandwidth and the sampling rate over which the sensor gathers information about the scene. A low spatial resolution multispectral (MS) and high spatial resolution panchromatic (PAN) imaging sensors are the systems usually used for earth change observation, each one having its own specific advantage. Most of the operating earth observation very high resolution (VHR) imagery (WorldView, QuicBird, GeoEye, and Orbview, etc.), it was very useful issues various RS problems such as image sharpening, land classification, change detection, and object identification, visualization purposes, etc. [1]. Besides that, PAN sensors provide an image in the visible band, which is characterized by high spatial information content well suited for intermediate scale mapping applications and urban analysis [2].

Nowadays, image fusion is also known as pan-sharpening, resolution merge, image integration, or multi-sensor data fusion. It is a useful tool in integrating a high-resolution PAN image with a low-resolution MS image to produce a highresolution multispectral image and a better understanding of the observed earth surface. In image fusion observed scene analysis and RS application, the observed scene information fusion can be available in the following cases [3]; data recorded by different sensors (multi-sensor image fusion), data recorded by the same sensor scanning the same scene at different dates (multi-temporal image fusion), data recorded by the same sensor operating in different spectral bands (multi-frequency image fusion), data recorded by the same sensor at different polarizations (multi-polarization image fusion), and data recorded by the same sensor located on platforms flying at different heights (multi-resolution image fusion). A Multi-sensor, multi-temporal, multi-resolution and multi-parameter image data from operational Earth observation satellites are available and therefore possibly give a complete view of observed objects [4] Recently, some researcher has addressed multi-temporal data fusion problem. Less publication focus on the multi-temporal image fusion and application has reported [3]-[9]. The exploitation of the multi-temporal information fusion is fundamental for change detection purpose but can be also valuable in image classification to improve the classification accuracy. And also study of fusing multiple images provided by heterogeneous image sensor has been proposed in many literatures with different methods, context, and purposes [10]. 
One of the major problems and limitations of existing image fusion techniques is the degradation in spectral quality (color distortion), which causes visual changes as well as spectral differences between the original and fused images. Another common problem is dependent of fusing quality on different wavelength sensitivity, and/or the preference of the operator, since the different operator or data type may lead to different fusion qualities. The effectiveness of different fusion techniques is examined to reduce color distortion in this study. Concerning various methods developed for image fusion for remote sensing, it is desirable to give a general assessment of the quality of the fused images from the viewpoint of practical use. Since the qualitative (visual) evaluation may contain subjective factor, possibly influence by personal preference, quantitative approaches are often required to prove the correctness of the visual evaluation

In this paper, we use fusion algorithm to assess the capability of fusion using multi-resolution analysis (MRA) and component substitution (CS) algorithm to observe of fused image for better quality in object identification. Also, we apply the following statistical approaches for standardizing and automating the evaluation process of the fused images. First, a qualitative assessment is made with the conventional visual inspection. Then, we examine the spectral quality between the original MS and the fused images.

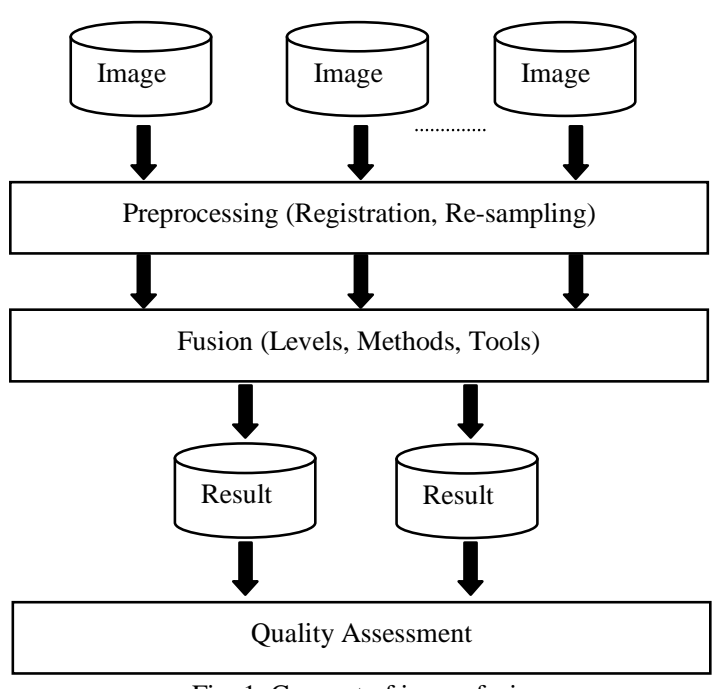

Fig. 1 Concept of image fusion

\section{MATERIALS AND METHODS}

The study site for this work is located in over the downtown of San Francisco, California (US) with geographical coordinates $122^{\circ} 23^{\prime} 1.08^{\prime} \mathrm{W}, 37^{\circ} 42^{\prime} 38.81^{\prime} \mathrm{N}$. San Francisco is located on the West Coast of the United States at the tip of the San Francisco Peninsula and includes significant stretches of the Pacific Ocean and San Francisco Bay within its boundaries (Fig. 2).
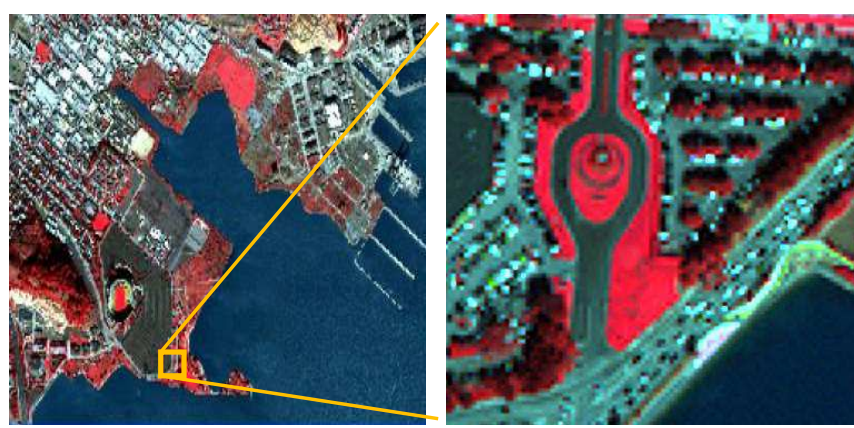

Fig. 2 Subset study area in downtown San Francisco, US

For this work, two temporal optical images acquired by QuickBird (QB) and WorldView-2 (WV) on 11 November 2007 and 9 October 2011, respectively, were used for investigating the performance of multi-sensor multi-multi temporal fusion. A QB image consists of one PAN and four MS with a spatial resolution of $0.7 \mathrm{~m}$ and $2.8 \mathrm{~m}$ at nadir and WV-2 image consists of one PAN and eight MS was placed on the altitude of $770 \mathrm{~km}$ with the revisit frequency of 1.1 days at 1 meter GSD (Ground Sample Distance) or less and 3.7 days at $20^{\circ}$ off-nadir or less (0.52 meter GSD). The optical temporal images used in this study were made available from Digital Globe, organized by IEEE GRSS in data fusion contest 2012. The characteristics of both images are summarized in Table 1.

TABLE I

CHARACTERISTICS OF VHR OPTICAL SENSORS

\begin{tabular}{|c|c|c|c|c|}
\hline Sensor & Band Name & Wavelength $(\boldsymbol{\mu m})$ & Resolution (m) & Date Acquisition \\
\hline \multirow{5}{*}{ QB } & B1(Blue) & $0.45-0.52$ & \multirow{4}{*}{$2.44-2.88$} & \multirow{9}{*}{11 November 2007} \\
\hline & B2(Green) & $0.52-0.60$ & & \\
\hline & B3(Red) & $0.63-0.69$ & & \\
\hline & B4(NIR) & $0.76-0.90$ & & \\
\hline & PAN & $0.45-0.90$ & $61-72 \mathrm{~cm}$ & \\
\hline \multirow{9}{*}{ WV-2 } & B1(NIR1) & $0.77-0.89$ & \multirow{4}{*}{2.07} & \\
\hline & B2(Red) & $0.63-0.69$ & & \\
\hline & B3(Green) & $0.51-0.58$ & & \\
\hline & B4(Blue) & $0.45-0.51$ & & \\
\hline & B5(R.Edge) & $0.70-0.74$ & \multirow{5}{*}{$52 \mathrm{~cm}$} & \multirow{5}{*}{9 October 2011} \\
\hline & B6(Yellow) & $0.58-0.62$ & & \\
\hline & B7(Coastal) & $0.40-0.45$ & & \\
\hline & B8(NIR2) & $0.45-0.48$ & & \\
\hline & PAN & $0.45-0.80$ & & \\
\hline
\end{tabular}


Significant spectral distortion in the fusion product image can occur due mainly to the wavelength extension of the new satellite PAN sensors. A major reason for the significant spectral distortion in image fusion is the wavelength extension of the new satellite PAN sensors. In image fusion techniques, it is important to include sensor spectral response information for the following reasons [11]: first, in order to preserve physical meaning of merged spectral bands, the sensor spectral response for each band has to be taken into account and second, image fusion techniques try to recover the image obtained by an ideal virtual sensor with the same spectral sensitivity of the MS and spatial resolution of the PAN sensor when during the fusion process. The spectral band's response curves of these two sensors are summarized in and Fig. 3. Table 2 shows the wavelength range and spatial resolution of different PAN sensors.

TABLE III

SPECTRAL RANGE OF DIFFERENT PAN SENSORS

\begin{tabular}{|c|c|c|}
\hline \multirow{2}{*}{ Sensor } & $\begin{array}{c}\text { Wavelength range } \\
(\boldsymbol{\mu m})\end{array}$ & $\begin{array}{c}\text { Spatial } \\
\text { resolution }(\mathbf{m})\end{array}$ \\
\cline { 2 - 3 } & $\mathbf{P A N}$ & 0.5 \\
\hline GeoEye-1 & $0.45-0.80$ & 0.7 \\
\hline QuickBird & $0.45-0.90$ & 1.0 \\
\hline Ikonos-2 & $0.45-0.90$ & 0.46 \\
\hline WorldView-2 & $0.45-0.80$ & 5 \\
\hline Spot 5 & $0.48-0.71$ & 10 \\
\hline EO1(ALI) & $0.48-0.69$ & 2.5 \\
\hline ALOS & $0.52-0.77$ & \\
\hline
\end{tabular}

A task for analyzing the image are divided into (1) Image correction and registration: The most important prerequisite for accurate data fusion is a precise geometric correction. In the image, fusion needs commons control point on both the input images since different images of the same area used together. The common geometric correction is an image to image registration. Registration can be done by various methods. One of the methods is an image to image registration, as seen in Fig. 4.

An image to image registration is translation and rotation alignment process by which two images of like geometry on the same geographic area [12]. In registration processing, the most accurate way is to register the input images separately by establishing a geometric relationship between the image and the ground using rigorous photogrammetric methods [13]. In this study, the first order polynomial transformation method is used for registration refinement of multispectral images by taking the WV multispectral image as the reference. A common ground control points (GCPs) on both images are selected carefully such that they produce an RMS error smaller than 0.5 pixels with respect to the pixel size of the fused WV image. The accuracy of image registration is usually conveyed in terms of root-mean-square (RMS) error, (2) Re-sampling: Next, we apply re-sampling, in which each data point (pixel) in the high-resolution base map is assigned a value based on the MS image pixels. In order to achieve a good fusion result, low spatial, spectral images should be resampled. At present, nearest neighbour resampling is often adopted which has some effects on the precision of new image.
QuickBird Relative Spectral Radiance Response
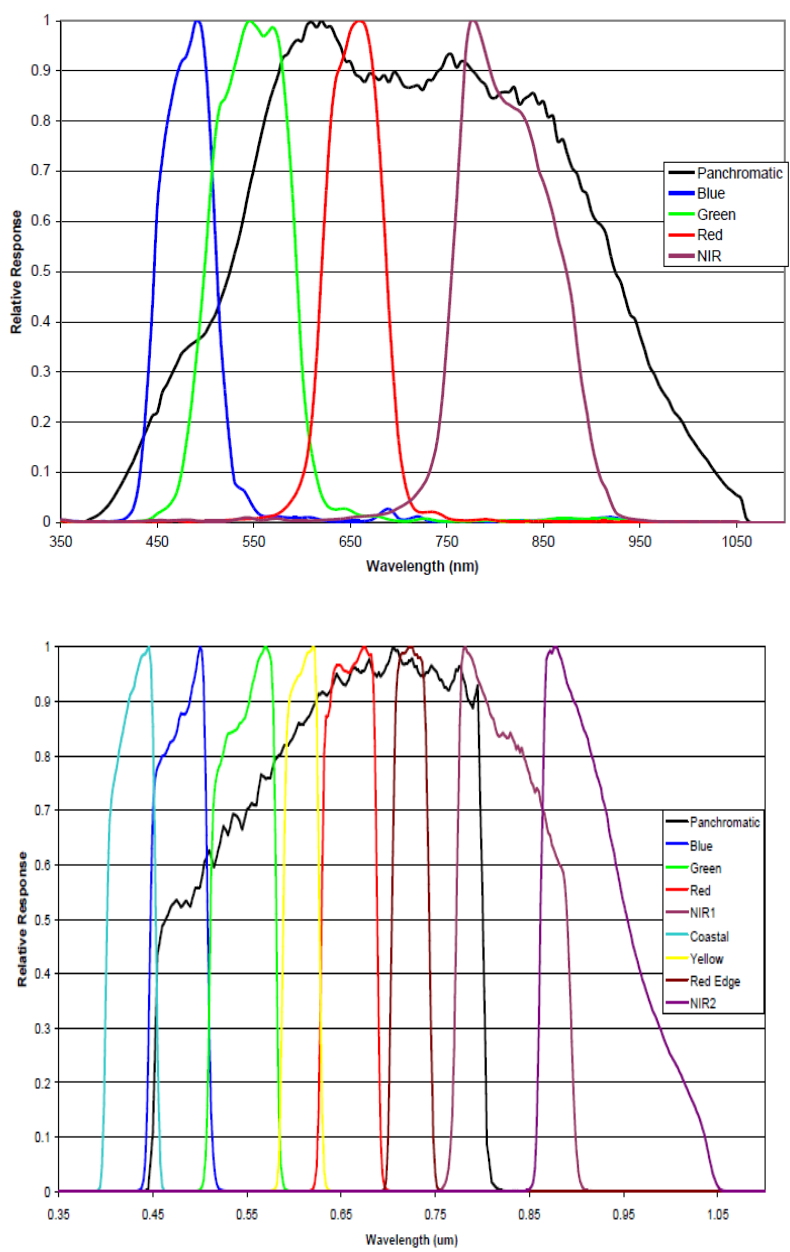

Fig. 3 Spectral response curves for QuickBird and WorldView-2

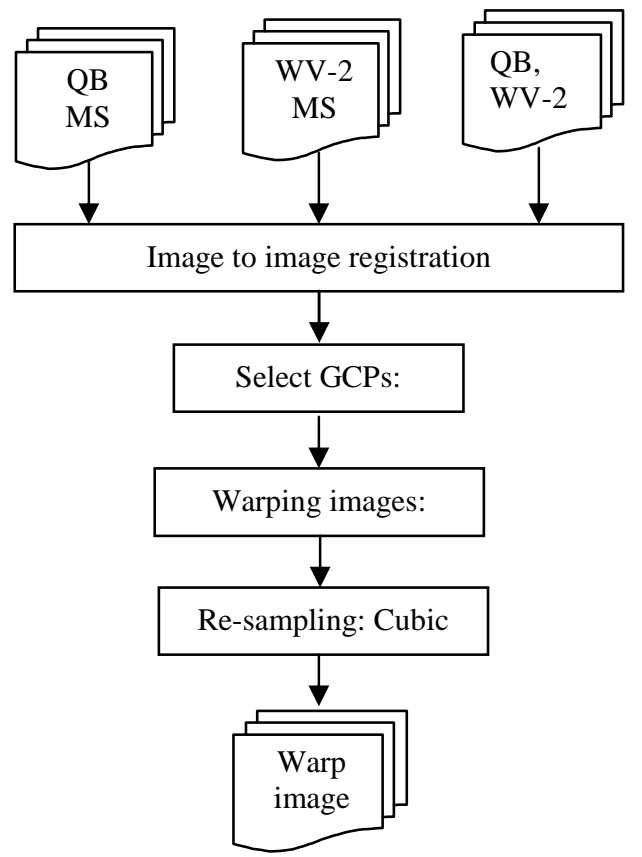

Fig. 4 Image to image registration processing

In this paper, an image fusion method is proposed with Cubic Convolution technique. In this way, PAN-MS images with $0.5 \mathrm{~m}, 2 \mathrm{~m}$, and $0.7 \mathrm{~m}, 2.8$ spatial resolutions are produced from original GeoEye-1 and QuickBird images, 
respectively (Fig. 3). The pixel size of WV PAN $(0.5 \mathrm{~m})$ is greater than that for QB PAN $(0.6 \mathrm{~m})$. Thus, in order to minimize the spectral difference, WV MS, QB MS and QB PAN imaging are used, after being re-sampled at $0.5 \mathrm{~m}$. To analyse the effect of different spatial resolution ratio images, re-sampling of the two images was done next as different resolution ratio to create a various set of images for fusion. The various resolution obtained due to such a re-sampling techniques are shown in Table 3 when the effective set of input image was generated for fusion using CS and MRA techniques, (3) Spatial resolution ratio: The spatial resolution ratio improvement can make a sharper image while preserving the spectral information contained in the fused images. Since PAN images usually have a better spatial resolution than the multispectral images of the same sensor, while the MS images provide spectral properties of the objects. In image fusion, spatial resolution ratio plays an important role in image fusion. The spatial resolution ratio can be defined as the ratio between the spatial resolution of the high-resolution PAN image and that of the lowresolution MS image [23]. The both of spatial resolution ratio of PAN to MS image was given in Table 3, (4) Band selection processing by the OIF: Band selection is a key step of fusion techniques. For this purpose, values of optimum index factor (OIF) are useful for designating the most favourable band combination according to their information [14]. Selecting of three bands for a color composite RGB (Red-Green-Blue) image is a tedious and time-consuming business since many combinations have to be tried when working with ratio images. The OIF technique simplifies that selection by the quantitative evolution of the scene statistics and avoids the time consuming of the visual analysis process of large numbers of potential R-G-B combinations. Since usually only three bands can be used for color composite (R-G-B), the spectral bands should be selected so as to project the MS information as efficiently as possible onto the final color image by calculating the OIF. Generally, a larger standard deviation of an image infers that it involves more information. Thus, the OIF is defined [15] as

$$
O I F=\sum_{i=1}^{3} \sigma_{i} / \sum_{j=1}^{3}\left|r_{j}\right|
$$

where $\sigma_{i}$ is the standard deviation of each of the three selected bands and $r_{j}$ is the correlation coefficients (CCs) between any pair formed by these bands (Table 4). From the original WV and QB, a total of 56 and 4 bands color combinations are produced and analyzed using the optimum index factor (OIF). The highest value of average OIF has been obtained for the band combination 3-5-7 and 2-3-4, both for WV and QB.

Multi-resolution analysis (MRA) is an approach based on Fast Fourier Transform (FFT)-enhanced intensity-huesaturation (IHS) transformation. It has been developed specifically for image merging that preserves spectral characteristics [6], [16]. The procedure of this method can be expressed as follows [16]-[17] (see Fig. 5). The re-sampled multispectral images are transformed from the RGB to IHS color space to obtain the intensity $(\mathrm{I})$, hue $(\mathrm{H})$, and saturation
(S) components, and low-pass filtering (LP) is applied to the intensity component.

TABLE IIIII

THE Set OF VARIOUs Spatial Resolution Ratio IMAges For Study

\begin{tabular}{|c|c|c|c|c|}
\hline $\begin{array}{l}\text { Test } \\
\text { case }\end{array}$ & Input Image & $\begin{array}{c}\text { Spatial } \\
\text { resolution } \\
(\mathbf{m})\end{array}$ & $\begin{array}{c}\text { Resolutio } \\
\text { n ratio }\end{array}$ & $\begin{array}{c}\text { Fusion } \\
\text { technique } \\
\text { s } \\
\end{array}$ \\
\hline I & \multirow{3}{*}{$\begin{array}{c}\text { QB_PAN + } \\
\text { QB_MS } \\
\text { QB_PAN + } \\
\text { WV_MS } \\
\text { WV_PAN + } \\
\text { WV_MS } \\
\text { WV_PAN + } \\
\text { QB_MS }\end{array}$} & $\begin{array}{c}\mathrm{PAN}=0.5 \\
\mathrm{~m}, \mathrm{MS}=2 \\
\mathrm{~m}\end{array}$ & $1: 4$ & CS, MRA \\
\hline II & & $\begin{aligned} \mathrm{PAN} & =10 \mathrm{~m} \\
\mathrm{MS} & =30 \mathrm{~m}\end{aligned}$ & $1: 3$ & CS, MRA \\
\hline III & & $\begin{array}{c}\mathrm{PAN}=0.5 \\
\mathrm{~m}, \mathrm{MS}=2 \\
\mathrm{~m}\end{array}$ & $1: 4$ & CS, MRA \\
\hline
\end{tabular}

After high-pass filtering, the panchromatic image is added to the LP filtered intensity component by means of inverse fast Fourier transform (FFT-1). Finally, inverse IHS transformation (IHS-1) is performed on the IHS image to create the fused image. The procedure of image fusion using the FFT-enhanced IHS transform method as given [16]

- Register all images the Pan image and re-sample to the highest resolution (e.g. Ikonos $1 \mathrm{~m}$, QuickBird $0.61 \mathrm{~m}$ ) using cubic convolution.

- Transform the re-sampled MS image from the RGB to IHS color space to obtain the intensity (I ), hue (H), and saturation (S) components.

- Low-pass filtering of the intensity component, ILP, by designing an appropriate filter in the Fourier domain.

- After high-pass filtering, the Pan image is added to the LP filtered intensity component by means of inverse fast Fourier transform $\left(\mathrm{FFT}^{-1}\right)$.

- Add the high frequency filtered Pan image to the low frequency filtered intensity component, I, for the new intensity component, ILP.

- Finally, inverse IHS transformation $\left(\mathrm{IHS}^{-1}\right)$ is performed on the IHS image to create the fused image.

A Component substitution (CS) make use of a filtering process to extract the high-frequency details from the PAN image fall in the category of component substitution methods. The principle idea is to add the details of the PAN image into the MS images making use of some transformation. The spatial resolution of the MS image is enhanced by merging the high-resolution PAN image with the low spatial resolution MS bands. The main steps of the methods are the following:

- A GS transformation is performed by employing the simulated PAN as the first band.

- After swapping the first GS band with the highresolution PAN image

- The inverse GS transform is applied to form the pansharpened spectral bands. 


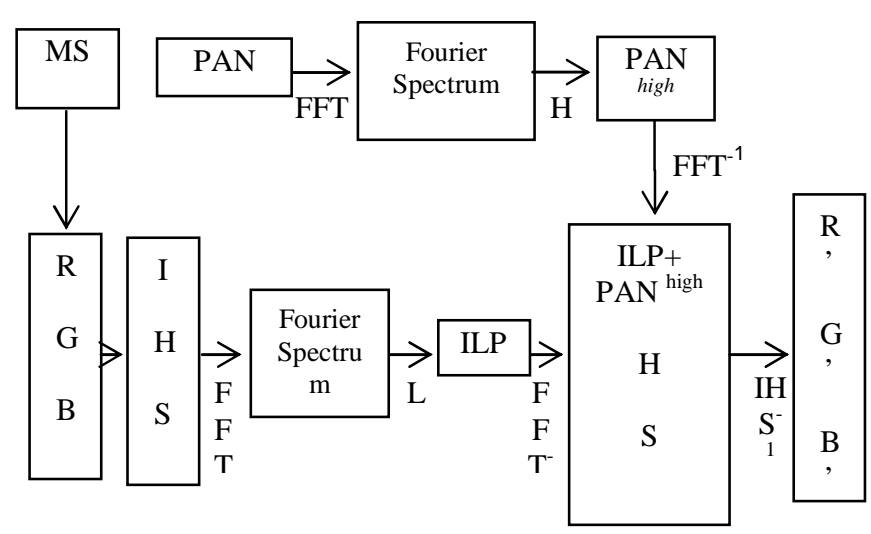

Fig. 5 FFT- enhanced IHS techniques based on MRA

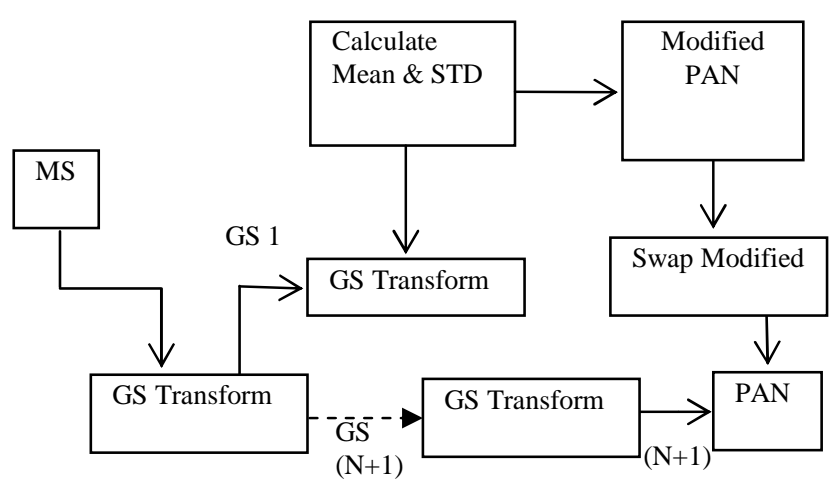

Fig. 6 GS Techniques based on CS

TABLE IVV

OIF INDEX FOR BANDS COMBINATION THE ORIGINAL IMAGES

\begin{tabular}{|c|c|c|c|c|}
\hline \multirow{2}{*}{$\begin{array}{c}\text { Satellite } \\
\text { Sensor }\end{array}$} & $\begin{array}{c}\text { Band } \\
\text { Combination }\end{array}$ & $\sum \boldsymbol{\sigma}_{i}$ & $\sum\left|r_{j}\right|$ & OIF \\
\hline \multirow{4}{*}{ WV } & $\mathbf{3 5 7}$ & $\mathbf{9 9 0 . 1 8}$ & $\mathbf{5 3 8 . 2 8}$ & $\mathbf{2 1 7 . 6 1}$ \\
\cline { 2 - 5 } & 257 & 956.4 & 481.75 & 210.55 \\
\cline { 2 - 5 } & 157 & 866.24 & 444.93 & 208.78 \\
\hline \multirow{4}{*}{ QB } & 123 & 830.91 & 534.01 & 207.65 \\
\cline { 2 - 5 } & 124 & 90.74 & 2.87 & 31.51 \\
\cline { 2 - 5 } & 134 & 163.49 & 2.17 & 75.16 \\
\cline { 2 - 5 } & $\mathbf{2 3 4}$ & 184.2 & 1.40 & $\mathbf{1 3 0 . 9 5}$ \\
\cline { 2 - 5 } & & & 2.08 & 79.52 \\
\hline
\end{tabular}

In the CS methods, so far many researchers have addressed the problem of multi-resolution and multitemporal image fusion for $\mathrm{RS}$ applications, proposing different methods such as Gram-Schmidt (GS), IntensityHue-Saturation (IHS) based fusion methods, Brovey transform based fusion and PCA based fusion.

The quality of fused image was assessed by means of some quality measures that can predict perceived image quality automatically. In this work, two types of quality assessment approaches are taken, namely qualitative (visual analysis) and quantitative methods. The visual analysis is based on the visual comparison of the color between the original MS and the fused image and that of the spatial details between the original PI and the fused image. These methods inevitably involve subjective factors and personal preference that can influence the result of the evaluation. While visual inspection has limitation due to human judgment, a quantitative approach based on the evaluation of "distortion" that is brought about by the noise added to the fused image is more desirable for mathematical modeling [18]. In this work, a validation method is proposed based on a quality criterion, namely, the relative average spectral error (RASE) [19]-[20] and relative dimensionless global error (ERGAS) [21]-[22] parameter. It is based on the root mean squared error (RMSE) [6] to estimation and chosen as a robustness criterion. This statistical parameter is often used for evaluation of fusion techniques. These parameters are defined as follows:

- Relative Average Spectral Error (RASE) is used to estimate the global spectral quality of the fused images

$$
R A S E=\frac{100}{M}\left[\frac{1}{n} \sum_{i=1}^{n} \operatorname{RMSE}^{2}\left(B_{i}\right)\right]^{1 / 2}
$$

Where $M$ is the mean radiance of the $n$ spectral bands (Bi) of the original MS bands; RMSE is the root mean square error computed as

$$
\operatorname{RMSE}\left(B_{i}\right)=\operatorname{Bias}^{2}\left(B_{i}\right)+S T D^{2}\left(B_{i}\right)
$$

- Relative Dimensionless Global Error in Synthesis (ERGAS) was proposed by Wald as a multi-modal index to characterize the quality of the process in terms of the normalized average error of each band of processed image. Increasing in ERGAS index may be the result of degradation in images due to the fusion process. ERGAS index for the fusion is expressed as follow

$$
E R G A S=100 \frac{d_{h}}{d_{l}}\left[\frac{1}{n} \sum_{i=1}^{n}\left(\frac{\text { RMSE }^{2}}{\text { mean }^{2}}\right)\right]^{1 / 2}
$$

These formulae can be used for comparing errors obtained from different methods, different cases and different sensors. Where $d h / d l$ is the ratio between the pixel sizes of the PAN and MS images (e.g., 1/4 for QB and WV data), and $\mu(i)$ is the mean of the $i$ th band. Since ERGAS is a measure of distortion, its value must be as small as possible.

For analysed information from multi-temporal assessment, the following combinations are employed here: (1) both PAN and MS images of November 2007 (QB-PAN, QBMS), (2) PAN of November 2007 and MS of October 2011 (QB-PAN,W-MS), (3) both PAN and MS images of October 2011 (WV-PAN, WV-MS), and (4) PAN of October 2007 and MS of November 2011 (WV-PAN,QB-MS). For each of these choices, both MRA and CS pan-sharpening methods are applied. 


\section{RESULTS AND DISCUSSION}

Fig. 7 and Fig. 8 shows the fused images obtained with the CS and MRA fusion methods for the four choices of band combinations.

TABLE V

QUALITY INDEX BASED ON CS FUSION WITH SPATIAL RESOLUTION RATIO 1:4

\begin{tabular}{|c|c|c|c|c|}
\hline \multirow{2}{*}{ Index } & \multicolumn{4}{|c|}{ Assessment Multi-Temporal Fusion } \\
\cline { 2 - 5 } & QB-PAN+ & QB-PAN+ & WV- & WV- \\
& QB-MS & WV-MS & $\begin{array}{c}\text { PAN+ } \\
\text { WV-MS }\end{array}$ & $\begin{array}{c}\text { PAN+ } \\
\text { QB-MS }\end{array}$ \\
\hline RMSE & 7.89 & 3.77 & 16.03 & $\mathbf{0 . 7 8}$ \\
\hline RASE & 0.54 & 0.19 & 0.83 & $\mathbf{0 . 0 5}$ \\
\hline ERGAS & 6.57 & 2.27 & 10.00 & $\mathbf{0 . 6 0}$ \\
\hline
\end{tabular}

TABLE VI

QUALITY INDEX BASED ON MRA FUSION WITH SPATIAL RESOLUTION RATIO 1:4

\begin{tabular}{|c|c|c|c|c|}
\hline \multirow{3}{*}{ Index } & \multicolumn{4}{|c|}{ Assessment Multi-Temporal Fusion } \\
\cline { 2 - 5 } & $\begin{array}{c}\text { QB-PAN+ } \\
\text { QB-MS }\end{array}$ & $\begin{array}{c}\text { QB-PAN+ } \\
\text { WV-MS }\end{array}$ & $\begin{array}{c}\text { WV-PAN+ } \\
\text { WV-MS }\end{array}$ & $\begin{array}{c}\text { WV- } \\
\text { PAN+ } \\
\text { QB-MS }\end{array}$ \\
\hline RMSE & $\mathbf{6 0 . 8 4}$ & 80.92 & 76.22 & 74.14 \\
\hline RASE & $\mathbf{3 . 4 5}$ & 3.76 & 4.24 & 3.65 \\
\hline ERGAS & $\mathbf{4 1 . 4 9}$ & 45.18 & 50.93 & 43.81
\end{tabular}

In the visual (quantitative) analysis, it is seen that $\mathrm{CS}$ fusion yields relatively sharp images for both PAN and MS images of October 2011 (WV-PAN, WV-MS) and PAN of October 2011 and MS of November 2007 (WV-PAN,QBMS). Other results show somewhat blurred results due to temporal changes. For MRA fusion, most of the color features for all combination has shown the preserve color with the original image. The visual analysis reveals that the quality both of WV-PAN,WV-MS, and WV-PAN, QB-MS are very good and quite comparable. However, the CS-based method gives a better result in terms of spatial quality (sharpness), whereas the MRA-based method yields better spectral quality, i.e., better color fidelity to the original MS images.

Table 5 and 6 have summarized the values of RMSE, RASE, and ERGAS indexes based on the CS and MRA approaches. Smaller parameter values (ideally zero values) indicate better preservation of the original information. The resulting index values obviously depend on the MS images chosen as reference. In the case of CS fusion, when the reference is the PAN of October 2011 and MS of November 2007 (WV-PAN, QB-MS), a better result is obtained as manifested in smaller values of RMSE, RASE and ERGAS. Since the higher of the value index denotes the degradation images.
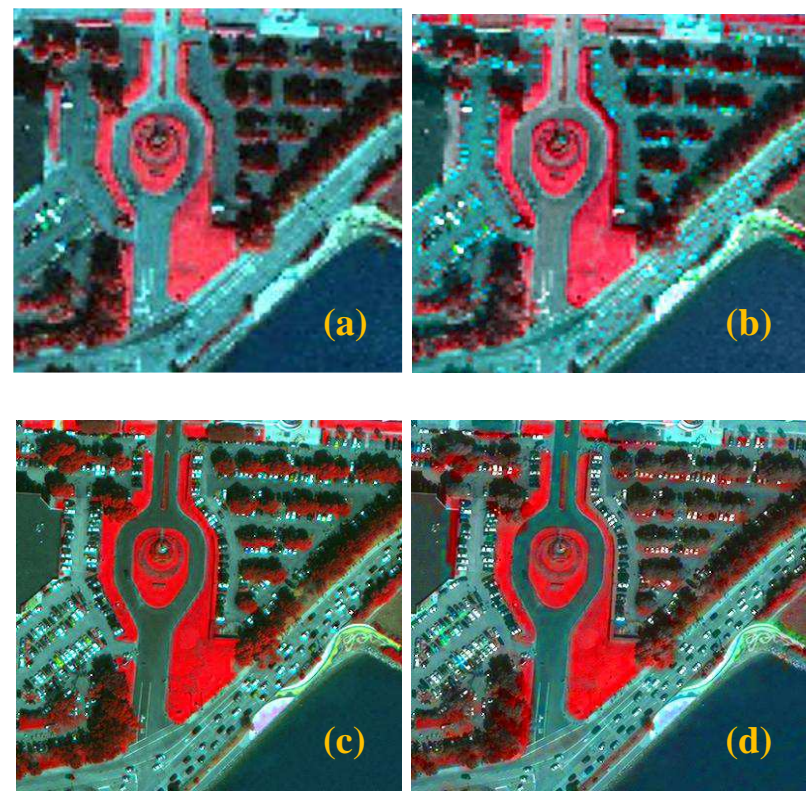

Fig. 7 Multi-temporal fusion assessment based on CS using (a) QBPAN+QB-MS (b) QB-PAN+WV-MS (c) WV-PAN+WV-MS (d) WVPAN+QB-MS
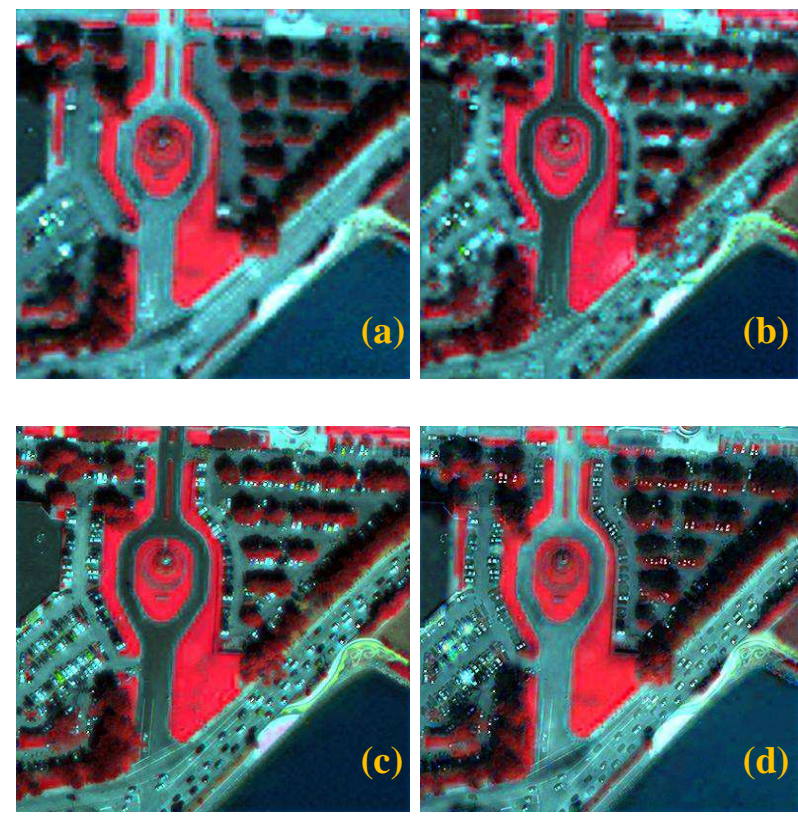

Fig. 8 Multi-temporal fusion assessment based on MRA CS using (a) QBPAN+QB-MS (b) QB-PAN+WV-MS (c) WV-PAN+WV-MS (d) WV$\mathrm{PAN}+\mathrm{QB}-\mathrm{MS}$

\section{CONCLUSIONS}

We have investigated the assessment of multi-temporal fusion by multi-resolution analysis (MRA) and component substitution (CS) algorithms. In both quantitative and qualitative results, it has been found that the CS based method leads to better spatial quality (sharpness), whereas the MRA based method better spectral quality (fidelity to the original color). In the future research, the methodology presented in this paper can be extended to include the multitemporal fusion of optical and synthetic aperture radar (SAR) images from satellite remote sensing. 


\section{ACKNOWLEDGMENT}

We would like to thank Digital Globe Inc. for providing the free download of Worldview-2 and QuickBird data from http://www.digitalglobe.com the anonymous reviewer for valuable comments and suggestions

\section{REFERENCES}

[1] Zeng, Y., Zhang, J., Van Genderen, J.L., Zhang, Y., Image fusion for land cover change detection, Int. J. Image and Data Fusion, 1(2), 193-215. 2010

[2] Chibani, Y., 2007, Integration of panchromatic and SAR features into multispectral SPOT images using the 'a trous' wavelet decomposition. Int. J. of Remote Sensing, 28, 2295-2307.

[3] Simone, G., Farina, A., Morabito., F.C., Serpico, S.B., Bruzzone, L., 2002. Image fusion techniques for remote sensing application, Information fusion, 3, 3-15.

[4] Zhu, L., Tateishi, R., 2006. Fusion of multi-sensor multi temporal satellite data for land cover mapping, Int. J. Remote Sensing, 27(5), 903-918.

[5] Cao, G., Hou, P., Ya-Qiu, J., Xianqiang, M., 2009. Fusion of features in multi-temporal SAR imagery to detect changes I urban areas, Int. J. Remote Sensing, 30(20), 5989-6001

[6] Ehler M, Klonus S, Astrand P.J, Rosso P. Multi-sensor for pansharpening in remote sensing, International Journal Image and Data Fusion.2010;1,1, 25-45

[7] Molch, K., 2010. Multi-sensor and multi-temporal fusion of VHR satellite imagery based on KIM, IEEE Geoscience and Remote Sensing Letter, 7(1).

[8] Kandrika, S., Ravinsankar, T., 2011. Multi-temporal satellite imagery and data fusion for improved land cover information ectraction, Int. J. Image and Data Fusion, 2,1, 61-73.

[9] Wurm, M., Taubenbock, H., Schardt, M., 2011. Object-based image information fusion using multi sensor earth observation data over urban areas, Int. J. Image and Data Fusion, 2,2, 121-147.

[10] Yuhendra, Alimuddin, I., Josaphat Tetuko, S.S., Kuze, H., 2012. Assessment of pan-sharpening methods applied to image fusion of remotely sensed multi-band data, Int. J. of Applied Earth Observation \& Geoinformation, 18,165-175.
[11] Otazu X, González M, Fors O, Núñez J. Introduction of Sensor Spectral Response Into Image Fusion Methods. Application to Wavelet-Based Methods, IEEE Transactions on Geoscience \&Remote Sensing.2005;43(10), 2376-2385.

[12] Chen, L., Lee, L., 1992. Progressive generation of control framework for image registration, Photogrammetric Engineering \& Remote Sensing, 58(9):1321-1328.

[13] Lee C, Bethel J, 2001 . Georegistration of Airborne Hyperspectral Image Data. IEEE Trans. on Geoscience and Remote Sensing. 39(7): 1347-1351.

[14] Choi M. A new intensity-hue-saturation fusion approach to image fusion with a tradeoff parameter, IEEE Trans. Geosciences Remote Sensing.2006;2 44, 1672-1682.

[15] Karathanassi V, Kolokousi P, Ionnidou S. A comparison study on fusion methods using evaluation indicators, Int. J. Remote Sensing. 2007; 28(10), 2309-2341.

[16] Ling Y, Ehler M, Usery E.L, Madden M. FFT-enhanced IHS transform method for fusing high-resolution satellite images, ISPRS Journal of Photogrammetry \& Remote Sensing. 2007; 61, 381-392

[17] Kumar U, Mukhopadhyay C, Ramachandra T.V. Pixel based fusion using IKONOS imagery, Int. J. Recent Trend in Engineering. 2009; 1(1), 173-175.

[18] Bovolo F, Bruzzone L, Capobianco L, Garzelli A, Marchesi S. Analysis of effect of pan-sharpening in change detection, IEEE Geoscine. Remote Sensing Letter. 2010; 7(1)

[19] Shah V. P, Younan N. H, and King R. L. An efficient pansharpening method via a combined adaptive PCA approach and contourlets. IEEE Trans. Geosciences Remote Sensing.2008; 4.

[20] Kumar U, Mukhopadhyay C, Ramachandra T.V. Pixel based fusion using IKONOS imagery, International Journal Recent Trend in Engineering.2009;1(1),173-175.

[21] Wang L, Cao X, Chen J. ISVR: an improve synthetic variable ratio method for image fusion, Geocarto International.2008; 23(2), 155165.

[22] Rahman M.M, Sri Sumantyo J.T, Sadek M.F. Microwave and optical image fusion for surface and sub-surface feature mapping in Eastern Sahara, International Journal Remote Sensing. 2010; 31(20), 5465-5480. 